\title{
Subcutaneous methotrexate for symptomatic control of severe recalcitrant psoriasis: safety, efficacy, and patient acceptability
}

REVIEW

This article was published in the following Dove Press journal:

Psoriasis: Targets and Therapy

5 May 2015

Number of times this article has been viewed

\section{Iviensan F Manalo' \\ Kathleen E Gilbert ${ }^{2}$ \\ Jashin $\mathrm{JWu}^{3}$}

'Medical College of Georgia, Georgia Regents University, Augusta,

GA, ${ }^{2}$ Indiana University School of Medicine, Indianapolis, IN,

${ }^{3}$ Department of Dermatology, Kaiser Permanente Los Angeles Medical

Center, Los Angeles, CA, USA

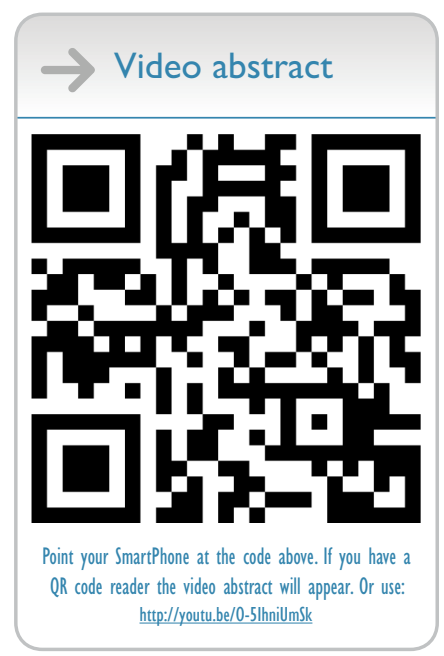

Correspondence: Jashin J Wu Department of Dermatology, Kaiser Permanente Los Angeles Medical Center, I5I5 North Vermont Ave, 5th floor, Los Angeles, CA 90027, USA

$\mathrm{Tel}+\mathrm{I} 3237834$ I 7 I

Fax + I 3237831629

Email jashinwu@hotmail.com
Background: Although oral methotrexate is an effective first-line traditional systemic therapy for psoriasis, the use of the subcutaneous form of methotrexate for the treatment of psoriasis has not been fully established.

Objective: This study is a literature review of the research related to the safety, efficacy, and patient acceptability of subcutaneous methotrexate for its application in the treatment of severe recalcitrant psoriasis.

Methods: Systematic literature searches were conducted of the PubMed, Ovid, and ClinicalTrials.gov databases.

Results: Only three relevant sources of literature were found studying subcutaneous methotrexate specifically in the context of psoriasis. Of these, only one clinical trial was found to directly study the use of subcutaneous methotrexate in psoriasis patients; however, results of this study have not been published. The other two literature sources involved a cost-effectiveness analysis and a literature review for subcutaneous methotrexate. Otrexup ${ }^{\mathrm{TM}}$ and Rasuvo ${ }^{\mathrm{TM}}$ are two particular single-use auto-injector modalities of subcutaneous methotrexate that are approved by the US Food and Drug Administration. The equivalents of Rasuvo available in countries outside of the USA are advertised as Metoject ${ }^{\circledR}$ or Metex ${ }^{\circledR}$. Much more research has been conducted on the use of subcutaneous methotrexate in rheumatoid arthritis patients.

Conclusion: There is a lack of original evidence-based studies evaluating the use of subcutaneous methotrexate specifically for the treatment of psoriasis. Based on the more extensively researched data on the safety, efficacy, and patient acceptability of subcutaneous methotrexate in rheumatoid arthritis patients, its application for the treatment of moderate-to-severe psoriasis is promising. More evidence-based studies on psoriasis subjects are needed to explore the practical application of subcutaneous methotrexate as a treatment option for severe recalcitrant psoriasis.

Keywords: self-administration, MTX, rheumatoid arthritis, Otrexup ${ }^{\mathrm{TM}}$, Rasuvo ${ }^{\mathrm{TM}}$

\section{Introduction}

Methotrexate (MTX) has been used for decades as the most commonly prescribed traditional systemic therapy worldwide for the treatment of psoriasis. ${ }^{1}$ Although its mechanism of action has not been fully elucidated, current evidence supports that it works by acting as both an antimetabolite (by inhibiting dihydrofolate reductase) and an immunomodulatory agent (by promoting adenosine release, thereby suppressing inflammation). ${ }^{2,3}$ Due to its anti-inflammatory quality, it is widely used as a treatment for various inflammatory diseases including but not limited to rheumatoid arthritis (RA), Crohn's disease, multiple sclerosis, psoriasis, and psoriatic arthritis. ${ }^{4}$ MTX has long been used as a standard of care to treat psoriasis and psoriatic arthritis at typically 
between 7.5 and $25.0 \mathrm{mg} /$ week in a single weekly dose or three divided doses separated by 12 hours every week, 5,6 although it has most extensively been studied for the treatment of RA. ${ }^{3}$ MTX is the disease-modifying anti-rheumatic first-line therapy for RA, and the majority of RA patients are treated in doses similar to those used for psoriasis. ${ }^{3,7}$ Various routes of administration exist for MTX: oral (PO), intravenous, subcutaneous (SC), and intramuscular for psoriasis, ${ }^{8,9}$ although the most commonly used route is still PO, largely due to convenience. ${ }^{10}$ The pharmacokinetics of SC administration for MTX were first examined in 1988, the same year that it was approved for the treatment of RA. ${ }^{11}$ Even though SC MTX has been more extensively studied in the context of RA, there has been a paucity of studies for understanding its application as a treatment of psoriasis. One particular paper published in August 2014 by Chiaravalloti and Strober presented the potential advantages and disadvantages of using self-administered SC MTX for the treatment of psoriasis based on a review of the literature. ${ }^{8}$ The aim of our paper is to present a systematic review regarding the efficacy, safety, and patient acceptability of SC MTX for the symptomatic control of severe recalcitrant psoriasis, with an update of additional research that has been conducted since the last review by Chiaravalloti and Strober and a focus on patient-oriented studies. Based on our findings, our secondary objective is to propose potential studies to advance our understanding on the use of SC MTX in the treatment of psoriasis.

\section{Methods}

A systematic electronic literature search using the terms "subcutaneous methotrexate" and "psoriasis" from the dates January 1, 1960 until December 31, 2014 was conducted of the PubMed, Ovid, US Food and Drug Administration (FDA), and ClinicalTrials.gov databases and search engines. Due to the limited literature published on the use of SC MTX in psoriasis, the aforementioned search was extended to SC MTX in the application of RA. Finally, a supplemental Internet search via the Google search engine for any remaining related publications was conducted using the keywords "subcutaneous methotrexate" and "psoriasis" with the constraints of being articles or announcements on human subjects only.

\section{Efficacy}

While the enteral route has been preferred due to its convenience, it can compromise the bioavailability of MTX, especially at higher doses, due to saturation of the gastrointestinal (GI) tract's absorptive capacity. ${ }^{12}$ At PO doses above $25 \mathrm{mg}$ /day the bioavailability of MTX becomes unpredictable, ${ }^{13}$ whereas the SC route demonstrates a linear and superior bioavailability in comparison., ${ }^{9,13-15}$

In 2008, Braun et al published the first and only clinical trial to provide clinical evidence for SC MTX's superior efficacy over PO MTX. The study population was MTXand biologic-naïve patients with active RA. The randomized controlled trial showed significant clinical improvement at 24 weeks with $15 \mathrm{mg} /$ week of SC MTX compared to $15 \mathrm{mg} /$ week of PO MTX, as defined by American College of Rheumatology criteria for 20\% (ACR20) and 70\% (ACR70) improvement outcomes. ${ }^{16}$ Although results from the study can be extrapolated to psoriasis, there has yet to be a study, as far as we are aware, that has published a head-to-head comparison of the clinical efficacy and safety of PO to SC MTX specifically for the treatment of psoriasis.

In the UK, a retrospective analysis was conducted of 103 RA patients who were switched from PO to SC MTX at a mean dose of $15 \mathrm{mg} /$ week due to either inefficacy or intolerance. Although both groups exhibited a significant improvement in their disease activity scores 3 months after switching, the group that switched due to intolerance notably displayed an even more significant improvement in disease activity. ${ }^{17}$

In October 2013, the FDA approved the first form of a single-dose disposable auto-injector modality for the onceweekly self-administration of SC MTX (Otrexup ${ }^{\mathrm{TM}}$, Antares Pharma, Ewing, NJ, USA) for RA, polyarticular juvenile idiopathic arthritis, and severe recalcitrant psoriasis. ${ }^{18}$ This form of SC MTX is available in dosage strengths of 7.5, $10.0,15.0,20.0$, and $25.0 \mathrm{mg}$. Subsequently, a randomized crossover head-to-head trial comparing the pharmacokinetics between the SC auto-injector and PO administration routes for MTX at doses of 10, 15, 20, and $25 \mathrm{mg}$ weekly in 47 RA patients demonstrated that the SC route achieved an increasingly higher, dose-proportional MTX exposure than the comparable PO dose, and the disparity continued to increase through doses of $25 \mathrm{mg} /$ week. The study also observed no increases in adverse events with SC MTX over its 8 -week period. ${ }^{15}$

Recently, the only clinical trial to most directly study the effect of SC MTX on psoriasis patients, as far as we are aware, has been completed. A Phase I randomized crossover clinical trial that concluded in October 2013 enrolled 35 subjects with moderate-to-severe psoriasis. Its aim was to determine the relative bioavailability of SC injection of $50 \mathrm{mg} / \mathrm{mL}$ MTX from a prefilled pen (Metoject ${ }^{\circledR}$, medac GmbH, Hamburg, Germany) versus intramuscular 
administration of $25 \mathrm{mg} / \mathrm{mL}$ MTX; however, the results of the study have yet to be published. ${ }^{19}$ This prefilled singledose disposable SC auto-injector pen produced by medac $\mathrm{GmbH}$ has the benefit of offering the widest dose range in a SC MTX modality, as it is available in ten different once-weekly dosage strengths that range from $7.5 \mathrm{mg}$ to $30.0 \mathrm{mg}$ in $2.5 \mathrm{mg}$ increments. It is most commonly available in numerous countries outside of the USA by the name of Metoject. medac $\mathrm{GmbH}$ also advertises the same product as Metex $^{\circledR}$ in several other European countries and Rasuvo ${ }^{\mathrm{TM}}$ in the USA. ${ }^{20}$ Only recently, in July 2014, has the FDA approved the use of Rasuvo. Similar to Otrexup, Rasuvo is approved for RA, polyarticular juvenile idiopathic arthritis, and severe recalcitrant psoriasis. ${ }^{21}$ In a single-center openlabel randomized two-period two-sequence single-dose crossover study of 62 subjects, the single-dose administration of Rasuvo demonstrated a higher relative bioavailability and fewer GI adverse events than PO MTX. ${ }^{22}$

\section{Safety}

The side effects of MTX are known to usually involve the GI system with common symptoms including nausea and anorexia. ${ }^{1}$ In gathering information regarding SC MTX's safety and tolerability profile, we examined the data for its safety profile in RA patients, since the dosages used to treat the majority of RA and psoriasis patients are similar. ${ }^{3}$ In a systematic literature review of 38 publications on the optimal dosage and route of administration of MTX in RA patients, observational data suggest fewer GI adverse events with parenteral administration of MTX.1,10,23

The first study to estimate the change in GI adverse events when converting from PO to SC MTX was recently published in June 2014. ${ }^{23}$ This retrospective survey of patients who had switched from PO to SC MTX measured responses on visual analog scales. Results of the survey showed that switching from PO to SC MTX resulted in significant improvements in the following GI adverse events: frequency of nausea, intensity of nausea, and frequency of discomfort. The only outcome not significantly reduced was frequency in vomiting. ${ }^{23}$ In another retrospective analysis of $80 \mathrm{RA}$ patients who had all converted from PO to SC MTX due to PO MTX's GI side effects, all the disease activity parameters and number of GI side effects had decreased at their first- and third-month visits, with no dropouts with SC MTX at the third-month visit. ${ }^{24}$ Although larger controlled studies are needed to further corroborate their results, these retrospective analyses indicate the potential for improvement in GI adverse events in patients who switch from PO to SC MTX.
The results of the previously mentioned trial by Braun et al that compared $15 \mathrm{mg} /$ week of PO to SC MTX showed that a superior clinical efficacy of SC MTX was not counterbalanced by a significant increase in frequency of adverse events. Both routes exhibited similar adverse events, and the frequency of adverse events was not significantly different, at $66 \%$ and $62 \%$ for the $\mathrm{PO}$ and SC routes, respectively. Despite these findings, however, more patients on SC MTX withdrew from therapy due to toxicity. ${ }^{16}$ These results were rather unexpected based on the earlier mentioned repeated findings from studies that the parenteral administration of MTX demonstrates fewer GI adverse events. No studies were found to specifically examine the effects of SC MTX on liver fibrosis. Moreover, the hepatotoxic effects of parenteral MTX have been controversial and mostly limited to small-scale studies. ${ }^{25-27}$

\section{Patient acceptability}

Patient acceptability is particularly significant in dictating adherence to therapy. As mentioned in the article by Chiaravalloti and Strober, a potential drawback to SC MTX is patients' fear of needles and/or the discomfort of selfinjecting; ${ }^{8}$ however, this doubt has been strongly reassured with the advent of the widely accepted biologics which require self-injection. ${ }^{15,16}$

In a large post-marketing surveillance study in Germany that examined the preference, satisfaction, usability, and tolerability of the SC self-administration of a ready-to-use prefilled syringe of high-concentration (50 mg/mL) MTX, $89.1 \%$ of patients endorsed that self-administration led to a feeling of more independence and improved quality of life $(83.6 \%) .{ }^{28}$ Although the majority of the study population were patients with RA, 59 (14.6\%) of the patients had psoriatic arthritis and the results of this patient-centered outcomes study can be generalized to psoriasis patients.

In a crossover study that examined RA patients' preference between the previously described prefilled pens and prefilled syringes of SC MTX at weekly doses of 15.0, 17.5, or $20.0 \mathrm{mg}$, the prefilled pen was generally favored with regard to patient use, acceptability, and satisfaction. ${ }^{29}$ Study nurses and physicians also recommended the prefilled pen due to convenience. ${ }^{29}$ Moreover, a study on Otrexup revealed that patients reported no or minimal pain at the administration site and a safe usability profile after proper training by site personnel and review of written instructions. ${ }^{30}$ This Phase II single-dose study was conducted in RA patients, 90.1\% of who had moderate-to-severe functional handicaps which may have affected manual dexterity. ${ }^{30}$ In a usability study on 
Rasuvo, 104 RA subjects reported consistently high degrees of easy performance, effectiveness, successful confirmation of delivery, and patient satisfaction. ${ }^{21}$ These studies suggest the benefits of a couple of nontraditional modalities (pen and auto-injector) for administering SC MTX to promote patient compliance.

The cost-to-efficacy ratio of a treatment option is also a significant factor to consider when evaluating its likelihood for patient adherence and suitability in clinical practice. A cost-effectiveness analysis shows the superior incremental cost-effectiveness ratio of SC MTX to cyclosporine, adalimumab, and infliximab in the treatment of moderate-to-severe psoriasis. ${ }^{31}$ A cost-effectiveness analysis conducted in Spain determined that the additional costs of Metoject compared to PO MTX would be compensated for by its improved effectiveness for RA in the Spanish Health System. ${ }^{32}$ Similarly, a cost-minimization analysis conducted in the UK demonstrated considerable savings for RA patients when switching from PO to SC MTX rather than to biologics. ${ }^{33}$

\section{Conclusion}

Multiple studies on SC MTX within the arena of RA point to the promising clinical benefits of SC MTX either as a monotherapy or combined with other therapies for psoriasis. However, there continues to be a scarcity of studies directly examining SC MTX for the management of psoriasis. So far, only one clinical trial has been found to study the effects of SC MTX in psoriasis patients; however, results of this trial have not been made available. ${ }^{19}$ Repeated findings from its use in numerous RA studies show that SC MTX has superior bioavailability at higher doses with no increase in frequency of GI adverse effects as compared to comparable doses via the PO route. ${ }^{15,16}$ It also has illustrated an improvement in quality of life, and favorable cost-to-efficacy ratio compared to biologics. ${ }^{32}$ Interpretation of these results suggests the extrapolated but not yet established superior clinical efficacy, safety, and patient acceptability of SC MTX for psoriasis patients. Hence, large-controlled studies must be done specifically in psoriasis subjects to further validate these interpretations. Although safety, efficacy, and patient acceptability are significant aspects in considering the integration of the SC route of MTX in the treatment algorithm for psoriasis, a more extensive pharmacoeconomics analysis of the cost-to-benefit ratio for its use would also prove to be valuable.

Table 1 presents proposed studies to address the research gaps that would help validate SC MTX's application in clinical practice for the treatment of psoriasis. As of the creation of this paper, no trials, as far as we are aware, have conducted
Table I Proposed studies for addressing research gaps in the utilization of subcutaneous methotrexate (MTX) for the treatment of psoriasis

I. Dose-ranging trials to optimize the safety and efficacy in utilizing subcutaneous MTX for the treatment of psoriasis

2. Clinical trials on the efficacy and adverse effect profile of using subcutaneous MTX in patients who have had an inadequate response or intolerance to oral MTX

3. Long-term safety, efficacy, and patient acceptability studies on utilizing subcutaneous MTX for the treatment of psoriasis

4. Studies comparing subcutaneous versus oral MTX's effects on liver fibrosis

5. Head-to-head comparisons of efficacy, safety, and patient acceptability between subcutaneous and oral MTX in the treatment of psoriasis

6. Efficacy of subcutaneous MTX for the treatment of recalcitrant psoriasis after failure of response to oral MTX

7. Efficacy of subcutaneous MTX for the treatment of recalcitrant psoriasis after failure of response to biologics

8. Efficacy of subcutaneous MTX when combined with other agents for the treatment of psoriasis

9. Quality-of-life outcomes in utilizing subcutaneous versus oral MTX

10. Cost-to-efficacy analyses of using subcutaneous MTX versus biologics

head-to-head comparisons between PO and SC administration of MTX for the management of psoriasis, although the call to address this insufficiency has already been previously mentioned. ${ }^{34}$ Proposed evidence-based studies that are needed to understand its application in clinical practice include: long-term and dose-ranging trials, efficacy and safety evaluations after switching from PO MTX, head-to-head comparisons with PO MTX and biologics, trials of SC MTX as a monotherapy or in combination with other therapies, quality-of-life studies, and cost-to-efficacy analyses.

This review reemphasizes the potential benefits of SC MTX that have been examined in RA. As suggested in studies on SC MTX in the RA population, ${ }^{10,34}$ SC MTX could be the bridge between insufficient response to PO MTX and attempting the much more expensive biologic therapies. Moreover, it could potentially replace PO MTX as a first-line systemic therapy for moderate-to-severe psoriasis due to its potential long-term cost-to-efficacy benefits while having better bioavailability with comparable tolerability. It could also maximize the symptomatic control of severe recalcitrant psoriasis when used in combination with biologics. Hence, we highly suggest that further comparison trials and studies for SC MTX be conducted directly examining psoriasis subjects on outcomes such as: Psoriasis Area Severity Index or Physician Global Assessment scores, patient quality-oflife questionnaires, adverse-event evaluations, and cost-toefficacy analyses. 


\section{Disclosure}

$\mathrm{Dr} \mathrm{Wu}$ has received research funding from AbbVie, Amgen, Coherus BioSciences, Eli Lilly, Janssen, Merck, Novartis, Pfizer, Regeneron, and Sandoz; he is a consultant for AbbVie, Amgen, Celgene, DUSA Pharmaceuticals, Eli Lilly, and Pfizer. The other authors do not have any conflicts of interest to disclose in this work. This work is exempted from review by an institutional review board and has not been presented previously.

\section{References}

1. Menter A, Korman N, Elmets C, et al. Guidelines of care for the management of psoriasis and psoriatic arthritis: section 4. Guidelines of care for the management and treatment of psoriasis with traditional systemic agents. J Am Acad Dermatol. 2009;61(3):451-485.

2. Cather J, Menter A. Novel therapies for psoriasis. Am J Clin Dermatol. 2002;3(3):159-173.

3. Weinstein GD, Jeffes EW III. Methotrexate. In: Koo JY, Levin EC, Leon A, Wu JJ, Gottlieb AB, editors. Moderate to Severe Psoriasis. 4th ed. Boca Raton, FL: CRC Press; 2014:151-168.

4. MedlinePlus. Methotrexate [web page on the Internet]. Bethesda, MD: National Institutes of Health United States National Library of Medicine; nd [updated May 15, 2014]. Available from: http://www.nlm.nih. gov/medlineplus/druginfo/meds/a682019.html. Accessed December 30, 2014.

5. Gottlieb A, Korman N, Gordon K, et al. Guidelines of care for the management of psoriasis and psoriatic arthritis: Section 2. Psoriatic arthritis: overview and guidelines of care for treatment with an emphasis on the biologics. J Am Acad Dermatol. 2008;58(5):851-864.

6. Kalb R, Strober B, Weinstein G, Lebwohl M. Methotrexate and psoriasis: 2009 National Psoriasis Foundation Consensus Conference. J Am Acad Dermatol. 2009;60(5):824-837.

7. Pincus T, Yazici Y, Sokka T, Aletaha D, Smolen JS. Methotrexate as the "anchor drug" for the treatment of early rheumatoid arthritis. Clin Exp Rheumatol. 2008;21(5 Suppl 31):S179-S185.

8. Chiaravalloti AJ, Strober BE. The use of self-administered subcutaneous methotrexate for the treatment of psoriasis. J Drugs Dermatol. 2014;13(8):929-931

9. Jundt JW, Browne BA, Fiocco GP, Steele AD, Mock D. A comparison of low dose methotrexate bioavailability: oral solution, oral tablet, subcutaneous and intramuscular dosing. J Rheumatol. 1993;20(11): 1845-1849.

10. Visser K, van der Heijde D. Optimal dosage and route of administration of methotrexate in rheumatoid arthritis: a systematic review of the literature. Ann Rheum Dis. 2009;68(7):1094-1099.

11. Balis F, Mirro J Jr, Reaman GH, et al. Pharmacokinetics of subcutaneous methotrexate. J Clin Oncol. 1988;6(12):1882-1886.

12. Tian H, Cronstein BN. Understanding the mechanisms of action of methotrexate: implications for the treatment of rheumatoid arthritis. Bull NYU Hosp Jt Dis. 2007;65(3):168-173.

13. Hoekstra M, Haagsma C, Neef C, Proost J, Knuif A, van de Laar M. Bioavailability of higher dose methotrexate comparing oral and subcutaneous administration in patients with rheumatoid arthritis. J Rheumatol. 2004;31(4):645-648.

14. Tuková J, Chládek J, Nemcová D, Chládková J, Dolezalová P. Methotrexate bioavailability after oral and subcutaneous dministration in children with juvenile idiopathic arthritis. Clin Exp Rheumatol. 2009;27(6):1047-1053.

15. Schiff MH, Jaffe JS, Freundlich B. Head-to-head, randomised, crossover study of oral versus subcutaneous methotrexate in patients with rheumatoid arthritis: drug-exposure limitations of oral methotrexate at doses $\geq 15 \mathrm{mg}$ may be overcome with subcutaneous administration. Ann Rheum Dis. 2014;73(8):1549-1551.
16. Braun J, Kästner P, Flaxenberg P, et al; MC-MTX.6/RH Study Group. Comparison of the clinical efficacy and safety of subcutaneous versus oral administration of methotrexate in patients with active rheumatoid arthritis: results of a six-month, multicenter, randomized, double-blind, controlled, phase IV trial. Arthritis Rheum. 2008;58(1):73-81.

17. Hameed B, Jones H. Subcutaneous methotrexate is well tolerated and superior to oral methotrexate in the treatment of rheumatoid arthritis. Int J Rheum Dis. 2010;13(4):e83-e84.

18. In brief: otrexup - a single-use auto-injector formulation of methotrexate. Med Lett Drugs Ther. 2014;56(1439):28.

19. medac $\mathrm{GmbH}$. Bioavailability in patient with psoriasis: Metoject prefilled pen. In: ClinicalTrials.gov [website on the Internet]. Bethseda, MD: US National Library of Medicine; 2014 [updated March 13, 2015]. Available from: https://www.clinicaltrials.gov/ct2/show/NCT02097 173? term=NCT02097173\&rank=1. NLM identifier: NCT02097173. Accessed January 2, 2015.

20. Metoject [homepage on the Internet]. Medac Pharma, Inc. Available at: http://www.metoject.com. Accessed January 29, 2015.

21. Rasuvo [drug label]. Chicago, IL: Medac Pharma, Inc.; 2014.

22. Pichlmeier U, Heuer K. Subcutaneous administration of methotrexate with a prefilled autoinjector pen results in a higher relative bioavailability compared with oral administration of methotrexate. Clin Exp Rheumatol. 2014;32(4):563-571.

23. Kromann C, Lage-Hansen P, Koefoed M, Jemec GB. Does switching from oral to subcutaneous administration of methotrexate influence on patient reported gastro-intestinal adverse effects? J Dermatolog Treat. Epup June 17, 2014.

24. Borman P, Demir G, Kaygısız F, Okumuş M. LETTER TO THE EDITOR Subcutaneous (SC) Methotrexate (MTX) is Better and WellTolerable than Oral MTX in Rheumatoid Arthritis Patients, Switched from Oral to SC Administration Due to Gastrointestinal Side Effects. Open Rheumatol J. 2014;8:18-19.

25. Vanscott EJ, Auerbach R, Weinstein GD. Parenteral methotrexate in psoriasis. Arch Dermatol. 1964;89:550-556.

26. Fry L. The treatment of psoriasis with parenteral methotrexate. $\mathrm{Br} J$ Dermatol. 1966;78(5):282-288.

27. Newman M, Auerbach R, Feiner H, et al. The role of liver biopsies in psoriatic patients receiving long-term methotrexate treatment. Improvement in liver abnormalities after cessation of treatment. Arch Dermatol. 1989;125(9):1218-1224.

28. Striesow F, Brandt A. Preference, satisfaction and usability of subcutaneously administered methotrexate for rheumatoid arthritis or psoriatic arthritis: results of a postmarketing surveillance study with a high-concentration formulation. Ther Adv Musculoskelet Dis. 2012; 4(1):3-9.

29. Demary W, Schwenke H, Rockwitz K, et al. Subcutaneously administered methotrexate for rheumatoid arthritis, by prefilled syringes versus prefilled pens: patient preference and comparison of the self-injection experience. Patient Prefer Adherence. 2014;8:1061-1071.

30. Freundlich B, Kivitz A, Jaffe J. Nearly pain-free self-administration of subcutaneous methotrexate with an autoinjector: results of a phase 2 clinical trial in patients with rheumatoid arthritis who have functional limitations. J Clin Rheumatol. 2014;20(5):256-260.

31. Puig L. Methotrexate: new therapeutic approaches. Actas Dermosifiliogr. 2014;105(6):583-589.

32. Crespo C, Brosa M, Galván J, et al. [Pharmacoeconomic analysis of Metoject $\left({ }^{\circledR}\right)$ in the treatment of rheumatoid arthritis in Spain.] Reumatol Clin. 2010;6(4):203-211. Spanish.

33. Fitzpatrick R, Scott D, Keary I. Cost-minimisation analysis of subcutaneous methotrexate versus biologic therapy for the treatment of patients with rheumatoid arthritis who have had an insufficient response or intolerance to oral methotrexate. Clin Rheumatol. 2013;32(11): 1605-1612.

34. Montaudié H, Sbidian E, Paul C, et al. Methotrexate in psoriasis: a systematic review of treatment modalities, incidence, risk factors and monitoring of liver toxicity. J Eur Acad Dermatol Venereol. 2011;25 Suppl 2:12-18. 
Psoriasis: Targets and Therapy

Dovepress

\section{Publish your work in this journal}

Psoriasis: Targets and Therapy is international, peer-reviewed, open access journal focusing on psoriasis, nail psoriasis, psoriatic arthritis and related conditions, identification of therapeutic targets and the optimal use of integrated treatment interventions to achieve improved system. Visit http://www.dovepress.com/testimonials.php to read real quotes from published authors.

Submit your manuscript here: http://www.dovepress.com/psoriasis-targets-and-therapy-journal 